\title{
High Dead Volume Pumps: Delay Time Reduction by Short-Term Low-Ratio Split Flow in LC/MS Applications
}

\author{
Eduard Rogatsky and Daniel T. Stein \\ General Clinical Research Center, Albert Einstein College of Medicine of Yeshiva University, Bronx, \\ New York, USA
}

The aim of our work was to develop a low-cost, simple and reliable solution to reduce LC/MS analysis time by compensating for limitations inherent to high dead volume standard HPLC pumps. In our approach, we utilized a temporary (sub-1 min) low ratio flow split $(\sim 1: 10)$ at $5 \mathrm{~mL} / \mathrm{min}$ pump flow before the column. During this short period, $90 \%$ of the entire pump flow is delivered to waste and used for fast pump/system equilibration. Although full-time flow splitting is widely used in capillary/nano applications (usually with high split ratios in the hundreds or thousands), to our knowledge, this is the first time that short-term low-ratio flow splitting has been used in conventional LC/MS applications. (J Am Soc Mass Spectrom 2007, 18, 245-247) (c) 2007 American Society for Mass Spectrometry

$\mathrm{H}$ undreds of thousands of HPLC gradient pumps (especially those manufactured in recent decades and optimized for $4.6 \mathrm{~mm}$ i.d. columns) have large dead volumes: $0.4 \mathrm{~mL}$ to $>1 \mathrm{~mL}$. In fast LC or LC/MS gradient applications, high dead volumes may cause a situation where the gradient front formed in the pump is delayed reaching the column [1]. Applications of high dead volume pumps with narrow bore columns, (e.g., $2 \mathrm{~mm}$ i.d., common in MS applications) which run at relatively low flow rates, result in slow equilibration (of the pump and then the column) and, therefore, in longer total analysis time. The general rule of thumb suggests 5 to 10 column volumes are required for equilibration. In practice, the impact of high pump dead volume or/and hardware design can considerably slow this process $[2,3]$. Pump/hardware related time delays are especially undesirable given the expense of LC/MS operations [4]. One of the possible solutions to overcome gradient delay problems arising from large pump dead volumes in LC/MS applications is isocratic elution. This, however, is only a partial solution when using small i.d. columns. If a step gradient is applied (for example 20\% B isocratic elution followed by $95 \%$ B for regeneration and back to $20 \%$ for equilibration), the delay problem will arise twice: during both regeneration and equilibration.

Although pump dead volume, in general, cannot be

Published online November 13, 2006

Address reprint requests to Dr. E. Rogatsky, General Clinical Research Center, Albert Einstein College of Medicine, Golding Bldg., Room G02, 1300 Morris Park Ave., Bronx, NY 10461, USA. E-mail: rogatsky@aecom.yu.edu physically reduced, the delay time can still be minimized. Several approaches are possible. The simplest one is based on inclusion of a high-pressure two position switching valve before the column. This plumbing arrangement allows optional pump purge to waste at high flow rate, while the LC column is bypassed (no flow) [5]. Advantages of this rapid equilibration approach are a simple and inexpensive design. However, the mobile phase composition at the end of the pump's purge to waste becomes significantly different compared with that remaining in the column. Some Type 1 silica-based C18 stationary phases are sensitive to such abrupt changes in mobile phase hydrophobicity. In addition, column flow interruption may be a serious disadvantage for high-temperature applications because of potential boiling of the mobile phase.

An alternative approach utilizes equilibration of a second pump while the first pump is running $[2,3]$. This approach is conducive to ultra-fast gradient applications since re-equilibration time is reduced by up to $75 \%$, and consistency is improved. The disadvantage of this strategy is a higher system cost invested into the second pump.

If an ultra-fast application is not the ultimate goal, implementation of a 10 port switching valve (instead of six port) in a two pump configuration enables operation of two identical columns [6]. The pumps do not need to be identical. Such a platform performs regeneration and equilibration of the second LC column, while injection and chromatographic separation is performed on the first column. This design greatly improves the throughput of LC/MS applications. Limitations of such two 

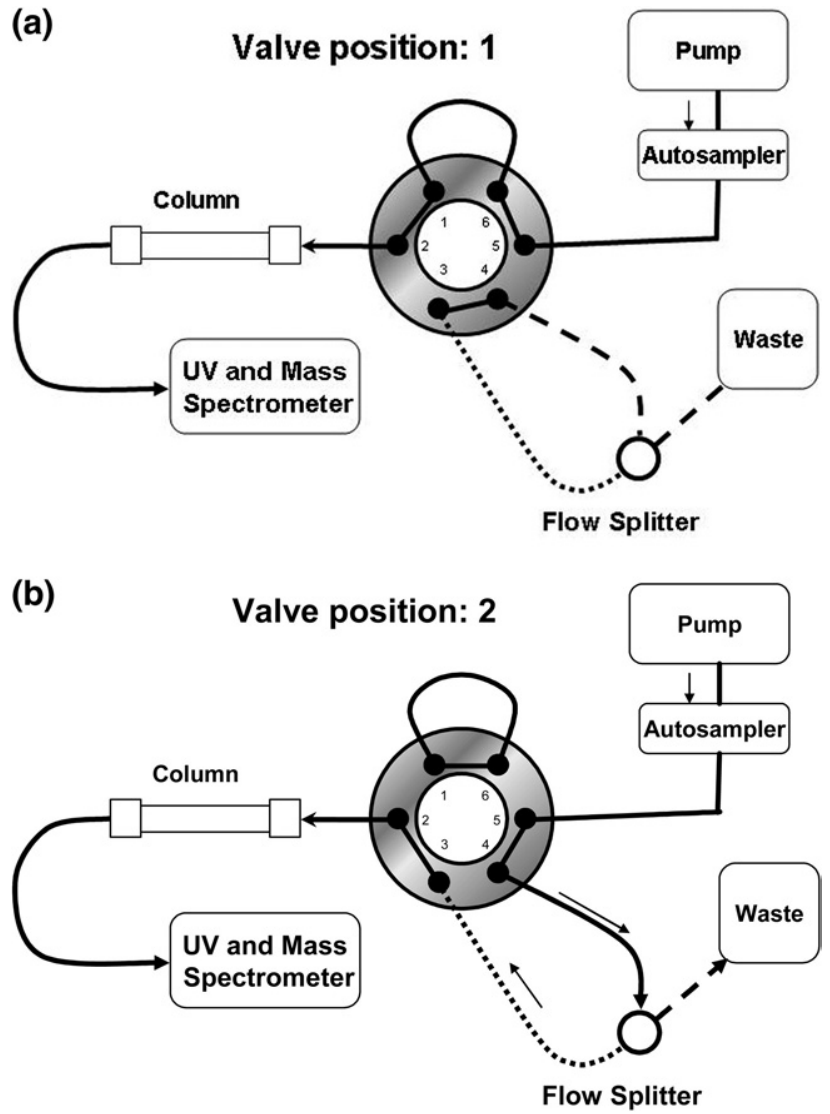

Figure 1. Hardware design (a) direct flow, (b) split flow.

pump/two column approaches are the requirement not only for HPLC software capability, but also the increased complexity of method development and the requirement for operator expertise. It should be emphasized that two pump configurations have reduced reliability compared with single pump designs because of the additional hardware. The efficiency and speed of column regeneration and equilibration will still be adversely affected if the second (regeneration) pump has a high dead volume.

Our approach utilizes a simple and reliable configuration based on one pump and one column. This design successfully avoids flow interruption and the risk of stationary phase shock. A high-pressure six port/two position switching valve is placed before the column. At valve position 1 (Figure 1a), the entire pump flow is delivered into the column (injection and elution steps). At valve position 2 (Figure 1b), pump flow is divided by the flow splitter. While a small part of the entire flow $(\sim 10 \%)$ is delivered into the column, the main part is directed to waste and serves to purge the pump. This system purge is only required during the first 0.6 to 0.9 min of column regeneration or equilibration steps. For initial development, we used an inexpensive low-pressure micro-splitter valve, P-451 from Upchurch Scientific (Oak Harbor, WA). Please note, this device is pressure/solvent viscosity dependent; therefore, the actual column flow rate (in split-flow mode) may vary somewhat with changes in mobile phase viscosity. We found that presetting the appropriate split ratio is time consuming. Use of fixed-ratio splitters (independent of solvent viscosity) can greatly simplify method development; however, such devices are much more expensive and contribute (by definition) higher system backpressure. For further development we used a preset 1:10 ratio flow splitter P\# 620-PR-10-10 available from ASI (El Sobrante, CA).

It should be emphasized that our purge approach efficiently reduces only the delay time caused by chromatographic instrumentation installed before the column. The dead volume of the remaining flow path can be reduced by the operator if necessary (see below).

To prove the efficiency of our proposed plumbing design, we compared the system equilibration time with and without flow splitting. During column regeneration and equilibration steps, the mass spectrometer was bypassed and column effluent was monitored by UV detection. The system was equilibrated with pure water during the first $4.99 \mathrm{~min}$, and than water containing $0.1 \%$ acetone was delivered from the $5.00 \mathrm{~min}$ time point. The typical UV-chromatogram of the nonsplit method (constant flow 0.2 and $0.5 \mathrm{~mL} / \mathrm{min}$ ) is given in Supplementary Material Figures 2 and 3, respectively (see Supplementary Material section, which can be found in the online version of the article). The total equilibration time (TET) was evaluated as the time from the start of delivery of mobile phase $\mathrm{B}(5 \mathrm{~min})$ till stabilization of $\mathrm{A}_{254}$ at the upper plateau. Surprisingly, even with the splitter bypassed and resulting simplified flow path, the full stabilization of $\mathrm{A}_{254}$ at the upper plateau at flow rate of $0.5 \mathrm{~mL} / \mathrm{min}$ was observed only after $30 \mathrm{~min}$; at $0.2 \mathrm{~mL} / \mathrm{min}$, full stabilization was obtained after $55 \mathrm{~min}$. These results contradict the general rule of thumb of 3 to 5 system volumes required for system equilibration. They are however consistent with other prior reports [2, 3]. If adequate equilibration for LC/MS applications is the goal, we conclude that a high dead volume pumps are not an appropriate choice.

The gradient delay and time to 95,99 and $99.5 \%$ of full equilibration is summarized in Table 1 . The gradient delay time for our application using the Agilent

Table 1. Gradient and equilibration delay times: effect of flow rate and split flow

\begin{tabular}{lcccc}
\hline \multicolumn{1}{c}{ Pump flow } & Gradient delay & $99.5 \%$ TET & $99 \%$ TET & $95 \%$ TET \\
\hline \hline $0.2 \mathrm{~mL} / \mathrm{min}$ & $3.16 \mathrm{~min}$ & $12.57 \mathrm{~min}$ & $9.48 \mathrm{~min}$ & $6.70 \mathrm{~min}$ \\
$0.5 \mathrm{~mL} / \mathrm{min}$ & $1.18 \mathrm{~min}$ & $4.57 \mathrm{~min}$ & $3.75 \mathrm{~min}$ & $2.73 \mathrm{~min}$ \\
$5 \mathrm{~mL} / \mathrm{min} / 1: 10 \mathrm{split}$ & $0.33 \mathrm{~min}$ & $0.92 \mathrm{~min}$ & $0.78 \mathrm{~min}$ & $0.64 \mathrm{~min}$ \\
\hline
\end{tabular}


quaternary pump (dead volume 0.8 to $1.1 \mathrm{~mL}$ ) was measured from the 5 min time point until the beginning of the absorbance rise from the basal plateau. For a 1:10 split (5 mL/0.5 mL/min), (Supplementary Material Figure 4 ), the delay time was $\sim 0.33$ min with a total equilibration time of less than $6.5 \mathrm{~min}$. Such a small delay time is comparable to delays of modern low-dead volume HPLC pumps. Ninety-five \% equilibration was achieved within $0.64 \mathrm{~min}$, which is more than 4 -fold faster than at the same column flow rate under nonsplit conditions. For regeneration or equilibration steps, our approach saves at least $4 \mathrm{~min}$ at $0.5 \mathrm{~mL} / \mathrm{min}(95 \%$ equilibration) and more than $10 \mathrm{~min}$ for $0.2 \mathrm{~min} / \mathrm{min}$ methods. Usually flow splitting is associated with large volumes of organic waste. In contrast, our approach (Supplementary Material Table S2) generates an insignificant additional volume of waste-only $2 \mathrm{~mL}$ per step.

Increasing of the split ratio, while reducing pump equilibration time, will result in lower flow through the column. In standard, noncapillary HPLC systems, post splitter dead volume can cause significant time delay at low flow rates. In our experimental set-up, we calculated that the post splitter tubing and UV dead volume was $\sim 60 \mu \mathrm{L}$. This added insignificant delay at 0.5 $\mathrm{mL} / \mathrm{min}$ column flow, but adds an additional $18 \mathrm{~s}$ delay at $0.2 \mathrm{~mL} / \mathrm{min}$. This delay can be reduced further by the use of smaller diameter HPLC tubing and low dead volume flow cells [7].

In summary, from the data presented we can see that with a conventional plumbing scheme (pump-columnmass spectrometer), the operator has only limited control over equilibration and regeneration since the speed of these steps is mainly hardware-related. Therefore, increasing throughput is usually performed by shortening the chromatographic separation time (e.g., by using a steeper gradient) which, in turn, may lead to increased matrix effects. An additional option, the use of shorter columns and/or smaller particle phases likely requires re-validation because of changes in analyte retention times. In contrast, in our application, increasing throughput is achieved by shortening pump delays in equilibration and regeneration steps and, therefore, the analyte retention time is unaffected. To achieve $99.5 \%$ of equilibration requires less than $1 \mathrm{~min}$, which is more than a 4-fold improvement compared to standard LC configuration. The short-term purge to waste is also beneficial in reduction of autosampler carryover, which in turn can improve LOQ. This simple modification of plumbing design can dramatically improve the usefulness of older generation high-dead volume but still reliable pumps for LC/MS analysis, and can overcome their "inherent" large delay time by saving 4 to $7 \mathrm{~min}$ per run while obtaining appropriate equilibration. However, the most efficient strategy of improving throughput of LC/MS analysis should be based on uncompromised implementation of low dead volume LC pumps.

\section{Acknowledgments}

This research was supported by grants from the American Diabetes Association, NIH/NCRR and NIDDK: MO1-RR12248 (GCRC) and R01 DK61644-01.

\section{References}

1. Majors, R. E. Fast and Ultrafast HPLC on Sub-3 $\mu \mathrm{m}$ Porous ParticlesWhere Do We Go from Here. LC/GC North Am. 2005, 23, 1248-1255.

2. Shellinger, A. P.; Stoll, D. R.; Carr, P. W. Accelerating Gradient Elution RPLC. Poster P-0356. Proceedings of the 28th International Symposium HPLC 2004; Philadelphia, PA, June, 2004.

3. Shellinger, A. P.; Stoll, D. R.; Carr, P. W. High Speed Reversed-Phase Gradient Elution Liquid Chromatography. J. Chromatogr. A 2005, 1064, 143-156.

4. Garcia, M. C.; Hogenboom, A. C.; Zappey, H.; Irth, H. Effect of the Mobile Phase Composition on the Separation and Detection of Intact Proteins by Reversed-Phase Liquid Chromatography-Electrospray Mass Spectrometry. J. Chromatogr. A 2002, 957, 187-1998.

5. Waters 2795 Operators Guide (P\# 71500279502) available on www. waters.com

6. Huber, U. High Throughput HPLC-Alternating Column Regeneration with the Agilent 1100 Series Valve Solutions. Agilent Technologies Application Note, publication number 5988-7831EN, 2002.

7. Woodman, M.; Zweigenbaum, J.; Schuette, S. Minimizing Resolution Losses in High Speed and High Resolution LC and LC/MS Separations. Poster 1270-17P. Proceedings of Pittcon 2006; Orlando, FL, March 2006. 